\title{
Independent trapping, manipulation and characterization by an all-optical biophotonics workstation
}

Hans-Ulrik Ulriksen

Jan Thøgersen

Soren R. Keiding

keiding@chem.au.dk

Ivan R. Perch-Nielsen

Jeppe S. Dam

\section{Darwin Z. Palima}

\section{Henrik Stapelfeldt \\ henriks@chem.au.dk}

\section{Jesper Glückstad \\ jesper.gluckstad@fotonik.dtu.dk}

Department of Physics and Astronomy, University of Aarhus, 8000 Aarhus C, Denmark

Department of Chemistry, University of Aarhus, 8000 Aarhus C, Denmark

Department of Chemistry, University of Aarhus, 8000 Aarhus C, Denmark

Interdisciplinary Nanoscience Center (iNANO), University of Aarhus, 8000 Aarhus C, Denmark

DTU Fotonik, Department of Photonics Engineering, Technical University of Denmark, 4000 Roskilde, Denmark

DTU Fotonik, Department of Photonics Engineering, Technical University of Denmark, 4000 Roskilde, Denmark

DTU Fotonik, Department of Photonics Engineering, Technical University of Denmark, 4000 Roskilde, Denmark

Department of Chemistry, University of Aarhus, 8000 Aarhus C, Denmark

Interdisciplinary Nanoscience Center (iNANO), University of Aarhus, 8000 Aarhus C, Denmark

DTU Fotonik, Department of Photonics Engineering, Technical University of Denmark, 4000 Roskilde, Denmark

Optical trapping has enabled a multitude of applications focusing, in particular, on non-invasive studies of cellular material. The full potential of optical trapping has, however, not yet been exploited due to restricted access to the trapped samples, caused by high numerical aperture objectives needed to focus the trapping laser beams. Here, we use our recently developed biophotonics workstation to overcome this limitation by introducing probing and spectroscopic characterization of optically trapped particles in a side-view geometry perpendicular to the trapping beams rather than in the traditional top-view geometry parallel to the trapping beams. Our method is illustrated by CARS and fluorescence spectroscopy of trapped polystyrene beads. The side-view geometry opens intriguing possibilities for accessing trapped particles with optical as well as other types of probe methods independent from the trapping process. [D0I: 10.2971/je0s.2008.08034]

Keywords: optical trapping and manipulation, coherent anti-Stokes Raman scattering, fluorescence spectroscopy, optical systems

\section{INTRODUCTION}

Optical tweezers have caused a revolution in trapping and manipulation of nano- and micrometer sized particles using strongly focused laser beams [1]-[3]. This has enabled a multitude of applications focusing, in particular, on non-invasive studies of cellular material. In principle it is quite simple why optical techniques based on trapping and manipulating nanoand micron sized samples have not yet reached their full potential and become a standard tool in bio-molecular laboratories. Using a tightly focused laser beam to trap the samples, the working distance to the object becomes very small, typically only a few tenths of a millimetre. Consequently, the trapped sample is only accessible through the microscope objectives used to focus the trapping laser. In practise, this limits characterization and imaging of the sample to a top-view geometry parallel to the trapping laser propagation axis. Thus, although the combination of optical trapping and spectroscopic characterization has been investigated previously, they were mostly limited to configurations where optical access to the trapped samples is through the optics of the trapping laser beams. Raman spectroscopy of optically trapped samples was introduced by Ajito and Torimitsu [4] and by Xie et. al. [5], the latter using the same diode laser for trapping and Raman excitation. More recently, Chan et. al. [6] and Shi et. al. [7] used lasers and super-continuum sources to simultaneously trap and characterize samples based on CARS (coherent anti-Stokes Raman) spectroscopy. Raman techniques have also been used for direct imaging of optically trapped cells. Creely et. al. [8] used Raman imaging at specific vibrational frequencies corresponding to protein- and lipid bands to image human T-cells. The acquisition time of the Raman spectrum was several minutes and again a high numerical aperture was required for the holographic optical tweezers. In these cases, the high numerical aperture objectives needed to focus the trapping laser beams restricts the access to the trapped samples, which also restricts the potential utility of the combined user-controlled manipulation and monitoring. An alternate configuration uses opposing optical fibers to deliver counterpropagating beams to create an optical trap that allows access to the trapped particle for independent monitoring along an orthogonal axis [9]-[11]. However, a fiber pair generates only a single trap whose manipulation capability is restricted to simple axial translation within the highly con- 
stricted operating region between the fiber tips. Scaling these systems to multiple traps capable of independent and arbitrary three-dimensional manipulation is nontrivial even based on purely geometrical constraints that can easily obscure the available orthogonal access.

In this work, we exploit our biophotonics workstation [12] to obtain autonomous access to the optically trapped samples and break away from restrictions of probing along the trapping laser axis. Multiple independently controllable counterpropagating beamlets delivered through low numerical aperture microscope objectives enable concurrent, yet independent, 3D manipulation of a plurality of particles within a large operating volume $[13,14]$. The geometry opens ample space for incorporating autonomous functional optical systems along the orthogonal axis for characterizing the trapped particles [15]. We show that optically trapped particles can be imaged and probed in a perpendicular or side-view geometry. We describe the system in Section 2 and demonstrate its capacity for simultaneous top and side-view optical microscopy of multiple trapped particles. The next sections then illustrate how optical systems can be tailored into the independent axis to characterize the optically trapped particles. Section 3 demonstrates autonomous fluorescence microspectroscopy of trapped samples while Section 4 demonstrates autonomous CARS microspectroscopy of trapped samples. Section 5 presents a summary and outlook.

\section{MULTIPARTICLE OPTICAL TRAPPING WITH CONCURRENT TOP- AND SIDE-VIEW MICROSCOPY}

The biophotonics workstation [12] used in this work is illustrated schematically in Figure 1. It employs an optical mapping from a beam modulation module to obtain reconfigurable intensity patterns corresponding to two independently addressable regions relayed to the sample volume where the simultaneous optical manipulation of a plurality of microobjects takes place. Fluid-borne microscopic particles are ushered in through a rectangular cuvette of high optical quality where they are trapped and steered in three dimensions using a real-time reconfigurable matrix of counter-propagating structured laser beams $(\lambda=1064 \mathrm{~nm})$. The counter- propagating geometry currently generates up to 100 powerful optical traps using well-separated objectives, which eliminates the need for high numerical aperture oil immersion objectives required in conventional optical tweezers. This generates a large field-of-view and leaves vital space for integrating other enabling tools for probing the trapped particles, such as linear and nonlinear microscopy or micro-spectroscopy. As is evident from Figure 1 the working distance, $\sim 1 \mathrm{~cm}$, of the objectives in the biophotonics workstation is much larger than the short working distance required in a standard optical tweezers setup.

The array of counter-propagating trapping-beams is easily aligned using a computer guided alignment procedure [16]. The spatial addressing of the expanded laser source is done in real-time through a high-speed computer controlled spatial light modulator that is integrated in the beam modulation

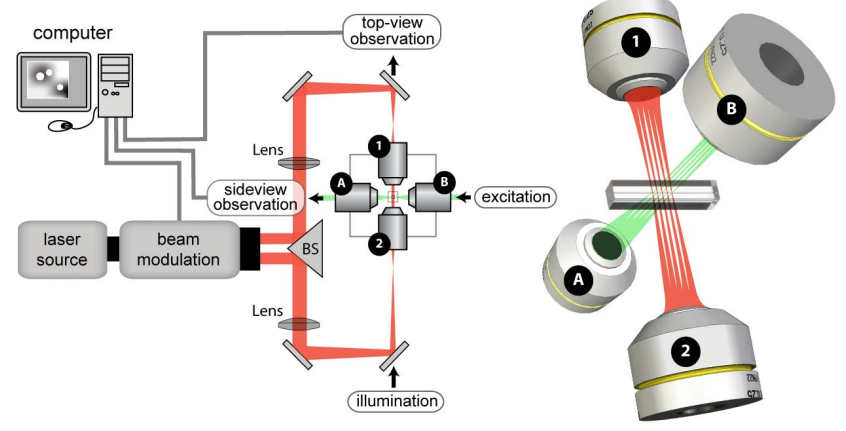

FIG. 1 Schematic of the experimental setup. The biophotonics workstation utilizes a single spatial light modulator illuminated by an expanded near-infrared laser source to generate an array of currently 100 fully reconfigurable counter-propagating laser-traps for $3 \mathrm{D}$ real-time optical trapping and manipulation. The counter-propagating geometry generates powerful optical traps even with well-separated objectives (numbered 1 and 2). This leaves space for integrating enabling spectroscopic and imaging tools with flow cells or incubators, for independently probing the trapped particles via objectives $A$ and $B$. In the present study the separation between objectives 1 and 2 is approximately $1 \mathrm{~cm}$.

module (see Figure 1). Through a computer interface, the operator can simply select, trap and move the desired object(s) with a mouse or joystick. Once trapped, arbitrary motion patterns can also be programmed for the sample(s) and complicated moving patterns of many independent samples can be orchestrated. The amount of particles being manipulated is only limited by the size of the working region (currently $\sim 70 \mu \mathrm{m}$ diameter). Examples of such manipulations are illustrated in Figure 2. Panels (a)-(f) demonstrate trapping and controlled rotation of a large polystyrene bead with a diameter of $40 \mu \mathrm{m}$. The bead is trapped by a large beamlet-pair and as a result of dragging an additional trap addressing the small bead attached to its surface the large bead can be precisely rotated around its axis. This manipulation feature could perform the function of a microscopic "workbench" for large cells or other microbiologic specimens. The images are obtained in both top- and side-view geometry (video online). In panels (g)-(l) we show a $3 \times 3$ array of small polystyrene beads $(\varnothing=4.5 \mu \mathrm{m})$ held in the optical trap. A simple moving pattern is defined allowing the $3 \times 3$ array to move. The $3 \times 3$ array can be viewed both in top-view and side-view geometry.

\section{OPTICAL TRAPPING WITH AUTONOMOUS FLUORESCENCE MICROSPECTROSCOPY}

The biophotonics workstation can host a variety of characterization tools along the independent orthogonal axis, such as linear and nonlinear microscopy and microspectroscopy. Initially, we use laser-induced fluorescence to characterize the samples in the trap. A frequency-doubled Nd:YAG laser pointer, $\sim 1 \mathrm{~mW}$ at $\lambda=532 \mathrm{~nm}$, induces fluorescence from a suspension of red fluorescence polystyrene beads $(\varnothing=$ $30 \mu \mathrm{m})$ held in the optical trap. Figure 3(a)-(d) shows a sequence of images obtained with a camera in the side-view geometry (video online). Using the computer mouse a trapped bead is gradually dragged into the exciting laser beam prop- 

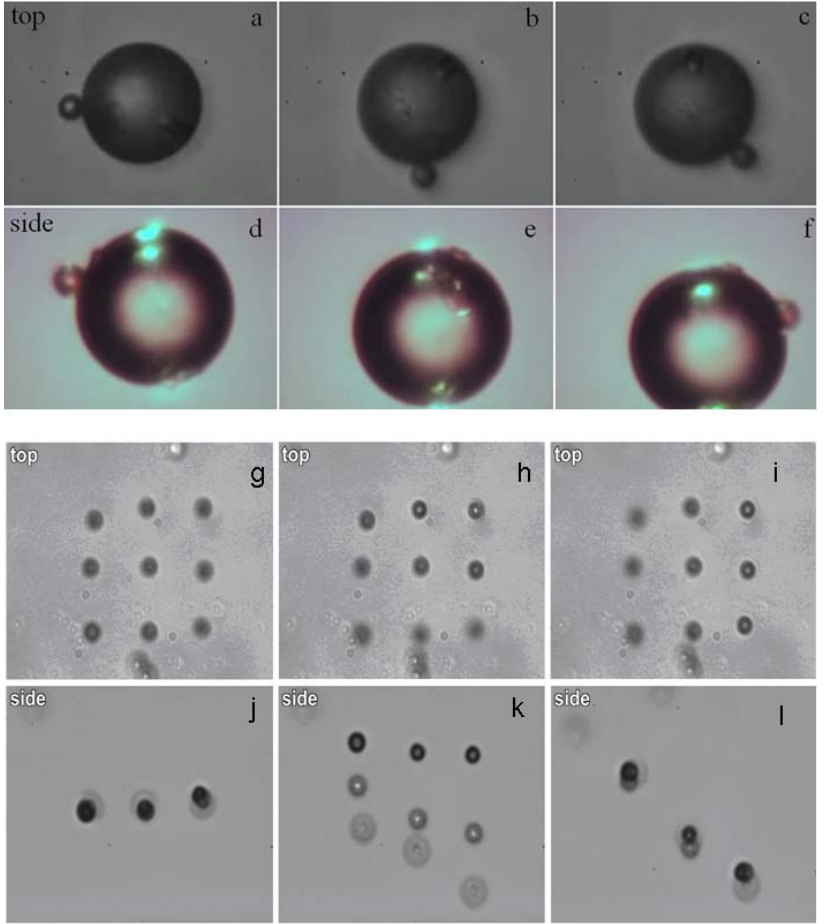

FIC. 2 Panels (a)-(f) shows a $\emptyset=30 \mu \mathrm{m}$ polystyrene bead trapped in the biophotonics workstation. A small defect on the side of the large bead was used to spin the large bead. Panels (a)-(c) show the spinning bead in top-view geometry and panels (d)-(f) show the bead in side-view geometry (videosize: 1.9 MB, format: avi, see Figza-f.avi). Panels (g)-(I) show nine $4.5 \mu \mathrm{m}$ polystyrene beads in a $3 \times 3$ configuration which are simultaneously manipulated. To change angle and amplitude of the tilt of the flat structure we use a joystick to alter the power ratio between the individual counterpropagating beams yielding the desired vertical displacements. The depth of focus for the top-view is quite large, so only with large displacements, as in panel (I), is the displacement visible through the defocusing visible on the three beads to the left in panel (i). The real-time online video shows the beads moving with speeds up to $20 \mu \mathrm{m} / \mathrm{s}$ (videosize: $2.9 \mathrm{MB}$, format: avi, see Fig2g-l.avi).

agating perpendicular to the image plane. When the bead is brought into overlap with the laser beam a strong fluorescence appears (Figure 3(d)), saturating the camera completely. When the polystyrene bead is outside the laser excitation beam it is still visible through its faint fluorescence induced by the room light. Figure 3(e), shows the unfiltered fluorescence spectrum obtained by replacing the camera, with a fibrecoupled spectrometer. The spectrum is composed of scattered light from the $532 \mathrm{~nm}$ laser and the strong fluorescence from the polystyrene beads extending from $500 \mathrm{~nm}$ to $700 \mathrm{~nm}$. In addition, a weak signal around $830 \mathrm{~nm}$ is observed originating from the diode laser used to pump the Nd:YAG-laser. The fluorescence results clearly show the advantage of the sideview geometry, enabled by the long working distance: Even the faint fluorescence signal, excited with a milliwatt laser pointer is easily detectable when the viewing direction is perpendicular to the strong trapping beams used.

\section{OPTICAL TRAPPING WITH AUTONOMOUS CARS MICROSPECTROSCOPY}

We now proceed to show how CARS spectra, and potentially CARS images, of trapped particles can be recorded in the

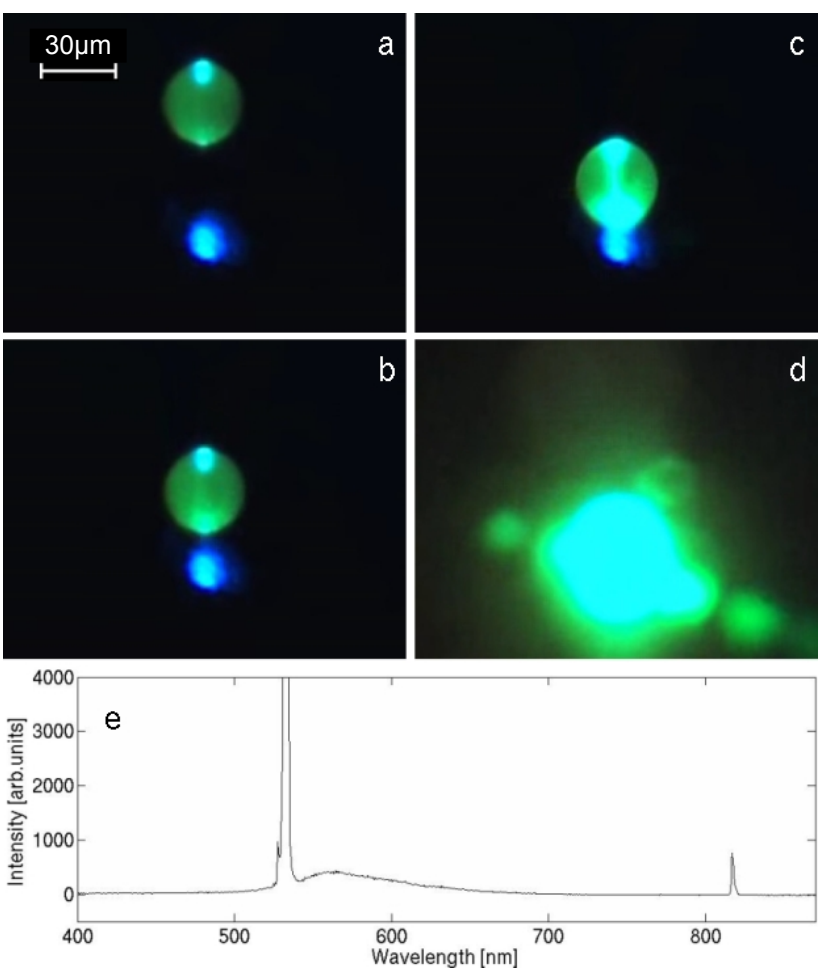

FIG. 3 Laser induced fluorescence from a dyed polystyrene bead trapped in the biophotonics workstation. In panel (a) the distance between the bead (green) and the $532 \mathrm{~nm}$ laser beam (blue) used to excite the fluorescence is approximately $30 \mu \mathrm{m}$. The faint fluorescence observable in panels (a)-(c) is induced by the room light. The trap is used to gradually move the bead closer to the $532 \mathrm{~nm}$ laser beam and in panel (d) they overlap giving rise to the fluorescence spectrum depicted in panel (e). Falsecolor contrasts the excitation from the fluorescence while maintaining their relative brightness (videosize: 1.4 MB, format: avi, see Fig3.avi).

side-view geometry. The ability to work with untagged or unstained samples is highly desirable for numerous reasons and it has been the driving force behind the rapid development of CARS microscopy and CARS-microspectroscopy since 1999 [17]-[19]. The CARS process is resonant scattering of coherently driven molecular vibrations. Consequently, it is both sensitive and chemically selective, and it gives similar information on the molecular composition of a sample as spontaneous Raman scattering. However, the coherent nature of the CARS process facilitates shorter acquisition times and furthermore reduces the detrimental effects of fluorescence from the sample. We drive the CARS process with a pump and a Stokes pulse using a single femtosecond laser and micro-structured crystal fibres $[20,21,28]$. The pump pulse is generated by spectral compression of a negatively chirped femtosecond pulse in a crystal fibre with its zero dispersion wavelength close to the laser wavelength of $800 \mathrm{~nm}$. This compresses the broadband femtosecond pulse to a near-transform limited picosecond pulse with a bandwidth of $1 \mathrm{~nm}$ corresponding to an order of magnitude increase in spectral brightness. The Stokes pulse is generated by launching an $800 \mathrm{~nm}$ femtosecond pulse into an optical fibre, dispersion shifted relative to the laser wavelength such that the Stokes pulse experiences negative group velocity dispersion. This results in the formation of a soliton and a gradual redshift of the soliton centre wavelength as the soliton propagates through the fibre [22]-[24]. By adjusting the laser power, the soliton can be continuously tuned from $800 \mathrm{~nm}$ to $1200 \mathrm{~nm}$, corresponding to Raman shifts up 
to $v_{R}=4000 \mathrm{~cm}^{-1}$.The frequency resolution in the present setup is determined by the spectral width of the compressed pump pulse and is $\Delta v_{R} \sim 15 \mathrm{~cm}^{-1}$. The collinear pump- and Stokes beams are focussed on the sample using a $\times 20$ objective and the phase matched anti-Stokes light is detected in the forward direction.

In Figure 4(a)-(d) we present a sequence of images showing a $10 \mu \mathrm{m}$ polystyrene bead in liquid water trapped in the biophotonics workstation. The images are obtained in the sideview geometry looking at the sample perpendicularly to the strong trapping beams. The red dot in the lower left side of the images corresponds to the two overlapping laser pulses at $\lambda_{P}=800 \mathrm{~nm}$ and $\lambda_{S}=1052 \mathrm{~nm}$, used to drive the CARS process. At these wavelengths the CARS process is resonant with the $\mathrm{C}-\mathrm{H}$ stretching modes around $3000 \mathrm{~cm}^{-1}$ in polystyrene and the resonant CARS signal appears at an anti-Stokes wavelength of $\lambda_{A S} \approx 645 \mathrm{~nm}$. In Figure 4(a), the trapped bead is located at the top of the image, barely visible as a faint red object. Since the trapping laser beams and the CARS laser beams are completely independent we can gradually move a trapped bead closer to the CARS lasers until they overlap. This is accomplished in Figure 4(d). When the bead is in the CARS laser beams a strong yellow signal is visible in the centre of the red spot. This yellow spot corresponds to the CARS signal at $645 \mathrm{~nm}$. The CARS signal is actually present in all four images. In addition to the resonant part of the CARS signal, there is also a non-resonant contribution to the CARS signal. Consequently, there is a weak non-resonant CARS signal from the solvent water molecules. The resonant CARS signal from polystyrene is several times stronger than the nonresonant background from water. Replacing the camera with a spectrometer, we can obtain the spectrum of the CARS signal from polystyrene as shown in Figure 4(e). In addition to the CARS signal at $3000 \mathrm{~cm}^{-1}$, we also measured the weaker CARS spectrum in the fingerprint region around $1600 \mathrm{~cm}^{-1}$ (not shown), where $\mathrm{C}-\mathrm{C}$ and $\mathrm{C}=\mathrm{C}$ vibrational modes contribute to the CARS spectrum [25]. The CARS spectral profile can either be calculated from known Raman lines, or if the trapped sample is of unknown molecular composition, the Raman lines can be extracted from the CARS profile using the maximum entropy method [26], allowing the line position to be disentangled from the non-resonant background. The solid line in Figure 4(e) is the calculated CARS profile based on the known Raman lines of Polystyrene and the wavelengths of the pump $\left(\lambda_{P}=800 \mathrm{~nm}\right)$ and the Stokes $\left(\lambda_{S}=1052 \mathrm{~nm}\right)$ pulses difference. We have obtained the CARS spectrum of polystyrene beads down to $5 \mu \mathrm{m}$ in diameter. For the smallest beads, the co-propagating CARS lasers will actually push the trapped particles slightly, requiring an adjustment of the trapping lasers. This can be minimized if the two CARS laser beams instead are counter propagating [27], which, in addition, will also reduce the non-resonant background.

\section{SUMMARY AND OUTLOOK}

We have demonstrated a system that decouples the optical trapping and manipulation from characterization and monitoring. This opens exciting possibilities for a broad range of techniques, where the samples can be characterized and ma-
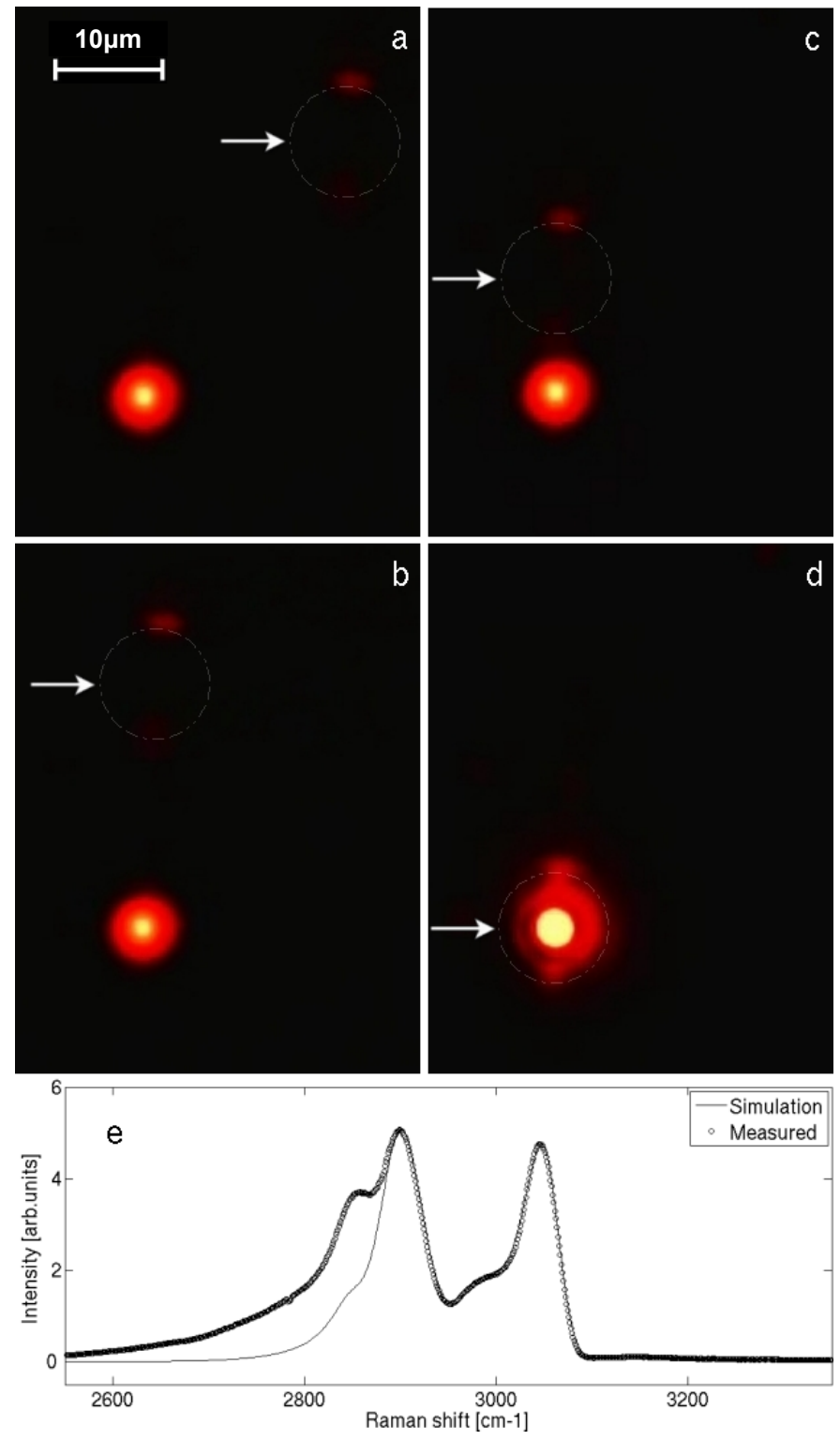

FIC. 4 CARS spectroscopy of trapped polystyrene beads $(\emptyset=10 \mu \mathrm{m})$. In panel (a) the barely visible bead, indicated with the arrow, is located far from the CARS lasers beams. The red spot in the lower left side of panel a is the non-resonant CARS signal from the water solvent and indicates the position of the CARS laser beams. Using the biophotonics workstation, the polystyrene bead is gradually moved closer to the CARS laser beams in panels (b) and (c) until the overlap in panel (d) (videosize: 2.5 MB, format: avi, see Fig4.avi). This gives rise to a strong CARS signal, resonant with the $\mathrm{C}-\mathrm{H}$ vibrational transitions in polystyrene. In panel (e) we show the CARS spectrum obtained when replacing the side-view camera with a spectrometer. From the known Raman spectrum of polystyrene we can simulate the CARS spectrum [26] and we observe good agreement between the measured and calculated spectrum. The minor discrepancy observed below $2850 \mathrm{~cm}^{-1}$ is due to additional chirp in the Stokes laser pulse [27] caused by the microscope objectives used for the CARS laser beams. This additional chirp can be removed by pre-compensating for the dispersion in the side-view optics.

nipulated independently of the trapping laser beams, thus facilitating sorting based on the molecular characteristics, fluorescence tagging for example, of the samples. Not only the full range of optical techniques are possible. The long working distances, and the ability to independently control the position and, importantly the orientation of the samples could also enable the use of miniaturized NMR-miniprobes [29] or 
X-Ray/electron beam scattering techniques to characterize the trapped samples. Furthermore, with the power levels used in the biophotonics workstation, both the trapping lasers and the laser pulses used for CARS, untagged live biological samples can be investigated in vitro for prolonged periods.

\section{ACKNOWLEDGEMENTS}

This work was supported by the Carlsberg Foundation and the Danish Agency for Science, Technology and Innovation.

\section{References}

[1] D.G. Grier, "A revolution in optical manipulation" Nature 424, 810816 (2003).

[2] A. Ashkin, J.M. Dziedzic, J.E. Bjorkholm, and S. Chu, "Observation of A Single-Beam Gradient Force Optical Trap for Dielectric Particles" Opt. Lett. 11, 288-290 (1986).

[3] J. Glückstad, "Microfluidics - Sorting particles with light" Nature Materials 3, 9-10 (2004).

[4] K. Ajito, and K. Torimitsu, "Near-infrared Raman spectroscopy of single particles" Trends Anal. Chem. 20, 255-262 (2001).

[5] C.G. Xie, M.A. Dinno, and Y.Q. Li, "Near-infrared Raman spectroscopy of single optically trapped biological cells" Opt. Lett. 27, 249-251 (2002).

[6] J.W. Chan, H. Winhold, S.M. Lane, and T. Huser, "Optical trapping and coherent anti-Stokes Raman scattering (CARS) spectroscopy of submicron-size particles" IEEE J. Sel. Top. Quant. 11, 858-863 (2005).

[7] K.B. Shi, P. Li, and Z.W. Liu, "Broadband coherent anti-Stokes Raman scattering spectroscopy in supercontinuum optical trap" Appl. Phys. Lett. 90, 141116 (2007).

[8] C.M. Creely, G. Volpe, G.P. Singh, M. Soler, and D.V. Petrov, "Raman imaging of floating cells" Opt. Express 13, 6105-6110 (2005).

[9] C. Jensen-McMullin, H.P. Lee, and E.R.L. Lyons, "Demonstration of trapping, motion control, sensing and fluorescence detection of polystyrene beads in a multi-fiber optical trap" Opt. Express 13, 2634-2642 (2005)

[10] P.R.T. Jess, V. Garcés-Chávez, D. Smith, M. Mazilu, L. Paterson, A. Riches, C.S. Herrington, W. Sibbett, and K. Dholakia, "Dual beam fibre trap for Raman micro-spectroscopy of single cells" Opt. Express 14 , 5779-5791 (2006).

[11] C. Liberale, P. Minzioni, F. Bragheri, F. De Angelis, E. Di Fabrizio, and I. Cristiani, "Miniaturized all-fibre probe for three-dimensional optical trapping and manipulation" Nat. Photon. 1, 723-727 (2007).

[12] J. Glückstad, I.R. Perch-Nielsen, J.S. Dam, and D. Palima, "Biophotonics workstation" Proc. SPIE 6905, 69050A (2008).

[13] P.J. Rodrigo, V.R. Daria, and J. Glückstad, "Four-dimensional optical manipulation of colloidal particles" Appl. Phys. Lett. 86, 074103 (2005).
[14] N. Arneborg, H. Siegumfeldt, G.H. Andersen, P. Nissen, V.R. Daria, P.J. Rodrigo, and J. Clückstad, "Interactive optical trapping shows that confinement is a determinant of growth in a mixed yeast culture" FEMS Microbiol. Lett. 245, 155-159 (2005).

[15] I.R. Perch-Nielsen, P.J. Rodrigo, and J. Clückstad, "Real-time interactive $3 \mathrm{D}$ manipulation of particles viewed in two orthogonal observation planes" Opt. Express 13, 2852-2857 (2005).

[16] J.S. Dam, P.J. Rodrigo, I.R. Perch-Nielsen, and J. Glückstad, “Fully automated beam-alignment and single stroke guided manual alignment of counter-propagating multi-beam based optical micromanipulation systems" Opt. Express 15, 7968-7973 (2007).

[17] J.X. Cheng, and X.S. Xie, "Coherent anti-Stokes Raman scattering microscopy: Instrumentation, theory, and applications" J. Phys. Chem. B 108, 827-840 (2004).

[18] A. Zumbusch, G.R. Holtom, and X.S. Xie, "Three-dimensional vibrational imaging by coherent anti-Stokes Raman scattering" Phys. Rev. Lett. 82, 4142-4145 (1999).

[19] N. Dudovich, D. Oron, and Y. Silberberg, "Single-pulse coherently controlled nonlinear Raman spectroscopy and microscopy" Nature 418, 512-514 (2002).

[20] E.R. Andresen, V. Birkedal, J. Thogersen, and S.R. Keiding, "Tunable light source for coherent anti-Stokes Raman scattering microspectroscopy based on the soliton self-frequency shift" 0pt. Lett. 31, 1328-1330 (2006).

[21] E.R. Andresen, J. Thogersen, and S.R. Keiding, "Spectral compression of femtosecond pulses in photonic crystal fibers" Opt. Lett. 30, 2025-2027 (2005).

[22] F.M. Mitschke, and L.F. Mollenauer, "Discovery of the Soliton SelfFrequency Shift" Opt. Lett. 11, 659-661 (1986).

[23] J.P. Gordon, "Theory of the Soliton Self-Frequency Shift" Opt. Lett. 11, 662-664 (1986).

[24] E.R. Andresen, C.K. Nielsen, J. Thogersen, and S.R. Keiding, "Fiber laser-based light source for coherent anti-Stokes Raman scattering microspectroscopy" Opt. Express 15, 4848-4856 (2007).

[25] P. Atkins, and J. De Paula, Physical Chemistry 8th edition (0xford University Press 2006).

[26] E.M. Vartiainen, H.A. Rinia, M. Muller, and M. Bonn, "Direct extraction of Raman line-shapes from congested CARS spectra" Opt. Express 14, 3622-3630 (2006).

[27] J.X. Cheng, A. Volkmer, and X.S. Xie, "Theoretical and experimental characterization of coherent anti-Stokes Raman scattering microscopy" J. Opt. Soc. Am. B 19, 1363-1375 (2002).

[28] E.R. Andresen, H.N. Paulsen, V. Birkedal, J. Thogersen, and S.R. Keiding, "Broadband multiplex coherent anti-Stokes Raman scattering microscopy employing photonic-crystal fibers" J. Opt. Soc. Am. B 22, 1934-1938 (2005).

[29] A.P.M. Kentgens, J. Bart, P.J.M. van Bentum, A. Brinkmann, E.R.H. van Eck, J.G.E. Gardeniers, J.W.G. Janssen, P. Knijn, S. Vasa, and M. Verkuijlen, "High-resolution liquid- and solid-state nuclear magnetic resonance of nanoliter sample volumes using microcoil detectors" J. Chem. Phys. 128, 052202 (2008). 